\title{
A matrix trace inequality
}

\author{
Feng Zhang*, Jinli Xu \\ Department of mathematics, Northeast Forestry University, Harbin, China, 150040
}

*Corresponding Author: Feng Zhang, Department of mathematics, Northeast Forestry University, Harbin, China, 150040

Abstract: In this short paper, we give a affirmative answer on matrix trace inequalities for the product of positive semidefinite matrices under a condition.

Keywords: Matrix trace inequality Majorization Positive semidefinite matrix Contractive.

\section{INTRODUCTION}

We give some notations, Let $M_{m \times n}(C)$ be the space of all complex matrices of size $m \times n$, For $A \in M_{n}(C)$, the vector of eigenvalues of $A$ is denoted by $\lambda(A)=\left(\lambda_{1}(A), \lambda_{2}(A), \ldots, \lambda_{n}(A)\right)$ If $A$ is Hermitian , we arrange the eigenvalues of $A$ in nonincreasing order, $\lambda_{1}(A) \geq \lambda_{2}(A) \geq \ldots \geq \lambda_{n}(A)$.In addition $x \prec y$ means that $x=\left(x_{1}, x_{2}, \ldots, x_{n}\right)$ is majorized by $y=\left(y_{1}, y_{2}, \ldots, y_{n}\right)$ with $x_{1} \geq x_{2} \geq \ldots \geq x_{n}$ and $y_{1} \geq y_{2} \geq \ldots \geq y_{n}$, if we have $\sum_{i=1}^{k} x_{i} \leq \sum_{i=1}^{k} y_{i}(k=1, \ldots, n-1)$ and $\sum_{i=1}^{n} x_{i}=\sum_{i=1}^{n} y_{i}$. $x \prec_{w} y$ means that $x=\left(x_{1}, x_{2}, \ldots, x_{n}\right)$ is weak majorized by $y=\left(y_{1}, y_{2}, \ldots, y_{n}\right)$, if we have $\sum_{i=1}^{k} x_{i} \leq \sum_{i=1}^{k} y_{i}(k=1, \ldots, n)$

The purpose of this paper is to give the answer to the following problem which was given in the paper [1].

Problem1.1 For $A, B \in M_{n}(C)$ are positive semidefinite matrices and $K$ is contraction, the following inequality hold or not?

In fact,we easily find that the inequality isn't true if $A, B$ is noncommutative, For example, Let $A=\left[\begin{array}{cc}10 & 0 \\ 0 & 1\end{array}\right], B=\left[\begin{array}{cc}1 & 3 \\ 3 & 10\end{array}\right], K=\left[\begin{array}{ll}0 & 1 \\ 1 & 0\end{array}\right], \quad A B \neq B A$, then $|\operatorname{tr} A K B|=33>\operatorname{tr}(A C)=20$.

2 Main resluts

Lemma1 Denoted the eigenvalues of matrix $A$ by $\lambda(A)=\left(\lambda_{1}, \ldots, \lambda_{n}\right)$, then $\left\{\left|\lambda_{i}\right|\right\}_{i=1}^{n} \prec_{w} s(A)$. Specially,$|\operatorname{tr} A| \leq \sum_{i=1}^{n} s_{i}(A) \cdot$

$\operatorname{Lrmma} 2 A_{1}, \ldots, A_{m}$ are $n$-square complex matrices, then $s\left(\prod_{j=1}^{m} A_{j}\right) \prec_{w}\left\{\prod_{j=1}^{m} s_{i}\left(A_{j}\right)\right\}_{i=1}^{n}$. 
Theorem3 $A, B \in M_{n}(C)$ are positive semidefinite matrices and $K$ is contraction and $A B=B A$, then $|\operatorname{tr} A K B| \leq \operatorname{tr} A B$

Proof $A, B \in M_{n}(C)$ are positive semidefinite matrices and $A B=B A$, so $A B$ is positive semidefinite.

According to Lemma2, $s(B A K) \prec s(B A)=\lambda(B A)$.Then

$|\operatorname{tr} A K B|=|\operatorname{tr} B A K| \leq \sum_{i=1}^{n} s_{i}(B A K) \leq \sum_{i=1}^{n} s_{i}(B A)=\sum_{i=1}^{n} \lambda_{i}(B A)=\operatorname{tr} B A=\operatorname{tr} A B$

So the theorem is completed.

Corollary4 $H=\left[\begin{array}{cc}A & B^{*} \\ B & C\end{array}\right]$ is positive semidefinite, $A C=C A$, then for any integer $m$, $\left|\operatorname{tr} B^{m}\right| \leq \operatorname{tr}\left(A^{\frac{m}{2}} C^{\frac{m}{2}}\right)$.

\section{REFERENCES}

[1] F.Kittaneh, M.Lin,Trace inequalities for positive semidefinite block matrices, Linear Algebra Appl.524 (2017) 153-158

[2] K. Audenaert, Subadditivity of q-entropies for q>1, J. Math. Phys. 48 (2007) 083507.

[3] R. Bhatia, Matrix Analysis, GTM, vol.169, Springer-Verlag, New York, 1997.

[4] M. Lin, A completely PPT map, Linear Algebra Appl. 459 (2014) 404-410.

[5] M. Lin, Inequalities related to $2 \times 2$ block PPT matrices, Oper. Matrices 9 (2015) 917-924.

[6] A.W. Marshall, I. Olkin, B. Arnold, Inequalities: Theory of Majorization and Its Applications, 2nded., Springer, 2011.

[7] F. Zhang, Matrix Theory: Basic Results and Techniques, 2nd ed., Springer, New York, 2011.

Citation: Feng Zhang \& Jinli Xu (2019). A matrix trace inequality. International Journal of Scientific and Innovative Mathematical Research (IJSIMR), 7(8), pp. 1-2. http://dx.doi.org/ 10.20431/2347 -3142.0708001 Copyright: () 2019 Authors, this is an open-access article distributed under the terms of the Creative Commons Attribution License, which permits unrestricted use, distribution, and reproduction in any medium, provided the original author and source are credited. 